\title{
Low-Frequency Noise Assessment of Different Ge pFinFET STI Processes
}

\author{
Alberto V. de Oliveira, Student Member, IEEE, Eddy Simoen, Member, IEEE, Jerome Mitard, \\ Paula G. D. Agopian, Senior Member, IEEE, Joao Antonio Martino, Senior Member, IEEE, \\ Robert Langer, Liesbeth Witters, Nadine Collaert, Aaron Voon-Yew Thean, Senior Member, IEEE, \\ and Cor Claeys, Fellow, IEEE
}

\begin{abstract}
An experimental low-frequency noise (LFN) assessment of long channel Ge pFinFET devices fabricated in different shallow trench isolation (STI) processes is presented, taking into consideration devices with fin widths from $100 \mathrm{~nm}$ (planar-like) down to $20 \mathrm{~nm}$. In addition, the correlation among LFN parameters, hole mobility and threshold voltage, is also evaluated. The carrier number fluctuation $(\Delta N)$ model is confirmed as dominant mechanism for all studied Ge pFinFETs and there is no correlation with the used STI process. From the LFN, it is evidenced that the Coulomb scattering mobility mechanism plays an important role for STI-first process, resulting in a mobility degradation.
\end{abstract}

Index Terms-Ge pFinFET, low-frequency noise (LFN), shallow trench isolation (STI) first, STI last.

\section{INTRODUCTION}

$\mathbf{T}$ HE combination of germanium as a channel material, because of its superior hole mobility compared with silicon [1], and multiple gate structures, such as FinFETs, which present greater electrostatic coupling and better short channel effects compared with planar devices [2], [3], has been pointed out as a promising device approach for high performance applications.

The high mobility materials, such as Ge and III-V [4], have been extensively investigated, motivated by the mobility

Manuscript received June 3, 2016; revised July 27, 2016; accepted August 3, 2016. Date of publication August 17, 2016; date of current version September 20, 2016. This work was supported in part by CAPES under Grant BEX 10537/14-7 and in part by the frame of the imec Core Partner Program on Ge devices.

A. V. de Oliveira is with the University of Sao Paulo, São Paulo 05508-010, Brazil, also with Katholieke Universiteit Leuven, Leuven 3001, Belgium, and also with imec, Leuven 3001, Belgium (e-mail: avo.eng@gmail.com).

E. Simoen, J. Mitard, R. Langer, L. Witters, and N. Collaert are with imec, Leuven 3001, Belgium (e-mail: eddy.simoen@imec.be; jerome.mitard@imec.be; robert.langer@imec.be; liesbeth.witters@imec.be; nadine.collaert@imec.be)

P. G. D. Agopian is with Universidade Estadual Paulista, São João da Boa Vista 13876-750, Brazil, and also with the University of Sao Paulo, Sao Paulo 05508-010, Brazil (e-mail: agopian@1si.usp.br).

J. A. Martino is with the University of Sao Paulo, São Paulo 05508-010, Brazil (e-mail: martino@lsi.usp.br).

A. V.-Y. Thean was with imec, Leuven 3001, Belgium. He is now with the National University of Singapore, Singapore 117575 (e-mail: aaron.thean@imec.be).

C. Claeys is with imec, Leuven 3001, Belgium, and also with Katholieke Universiteit Leuven, Department of Electrical Engineering, Leuven 3001, Belgium (e-mail: c.claeys@ieee.org).

Color versions of one or more of the figures in this paper are available online at http://ieeexplore.ieee.org.

Digital Object Identifier 10.1109/TED.2016.2598288 degradation when high- $\kappa$ dielectrics compose the gate-stack [5]. However, in order to enable industrial manufacturing, the Si-platform integration must be taken into consideration for future devices. However, heteroepitaxy on a Si substrate may be prone to extensive defect generation, mainly misfit dislocation and threading dislocation (TD) [6], since there is a mismatch in lattice parameter and coefficient of thermal expansion between $\mathrm{Si}$ and $\mathrm{Ge}$ [7]. Moreover, the TD density is a key parameter to be reduced, since it strongly affects the device performance [8], [9].

In order to keep the TD density as low as possible, different techniques have been evaluated to grow a Ge layer, which can be either on top of a $\mathrm{Si}-\mathrm{Ge}$ strain relaxed buffer (SRB) [10] or directly on silicon [11]. The first process fabricates SRB layers in predefined shallow trench isolation (STI) region (referred to as STI-first) [12], where the SRB layer must be thick enough, i.e., with an aspect ratio higher than 3 , so that most of the TDs are trapped in the bottom part of the layer [13]. In another approach, a thick SRB layer $(\sim 1 \mu \mathrm{m})$ is grown on a $\mathrm{Si}$ wafer, followed by a thin Ge layer on the SRB, which can achieve a TD density in the SRB around $10^{6} \mathrm{~cm}^{-2}$ [14]. Subsequently, the fin is defined by an STI process, which in this case is called STI-last. A modified option is the growth of a thick Ge layer on the Si substrate, followed by a chemical mechanical polishing. The fin is also defined by the STI-last process [11]. It is worth mentioning that the growth of a thin Ge layer on the SRB results in a compressive stress, which can have a beneficial impact on the p-channel device performance [15].

Low-frequency noise (LFN) measurement has been systematically used in this paper, which is one important, powerful and nondestructive technique that allows evaluating in detail the gate dielectric and device channel quality [16].

This paper has the following sequence: Section II starts with the device characteristics, where the main dimensions and process data are presented. Section III describes how the LFN analysis has been done. Section IV shows both LFN parameters, hole mobility and threshold voltage behaviors, as a function of the fin width. Section V concludes with the correlation among the main studied parameters from Section IV in order to evaluate and identify the parameters that contribute to the hole mobility and the threshold voltage degradations. 
TABLE I

DeVice Dimensions for the DifFerent STI Processes

\begin{tabular}{|c|c|c|c|}
\hline & $\begin{array}{l}\text { STI first } \\
\text { strained }\end{array}$ & $\begin{array}{c}\text { STI last strained } \\
\text { using } \mathrm{SRB} / \mathrm{Si}\end{array}$ & $\begin{array}{l}\text { STI last relaxed } \\
\text { using } \mathrm{Ge} / \mathrm{Si}\end{array}$ \\
\hline $\mathrm{W}_{\text {fin }}(\mathrm{nm})$ & \multicolumn{3}{|c|}{20 to 100 (planar-like) } \\
\hline $\mathrm{L}_{\mathrm{G}}(\mathrm{nm})$ & \multicolumn{3}{|c|}{330 to 1,000} \\
\hline $\mathrm{H}_{\text {fin }}(\mathrm{nm})$ & 20 & 30 & 30 \\
\hline $\mathrm{N}$ & \multicolumn{3}{|c|}{4} \\
\hline $\mathrm{Si}_{1-\mathrm{x}} \mathrm{Ge}_{\mathrm{x}}$ & 75 & 70 & N.A. \\
\hline Gate stack composition & \multicolumn{3}{|c|}{$\mathrm{Ge} / \mathrm{Si}-\mathrm{cap} / \mathrm{HfO}_{2} / \mathrm{TiN} / \mathrm{W}$} \\
\hline
\end{tabular}

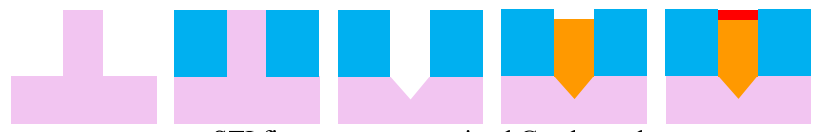

a - STI first process - strained Ge channel

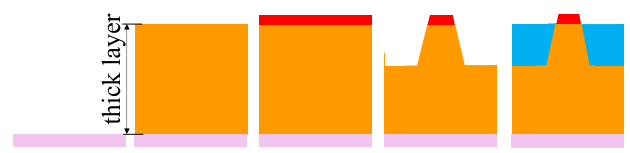

b - STI last process - strained Ge channel

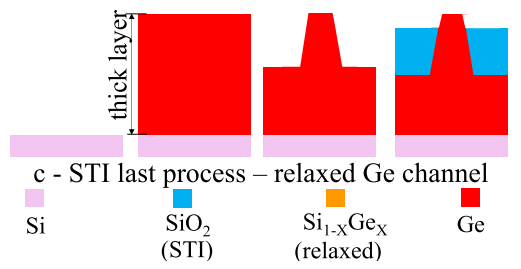

Fig. 1. Schematic of the three different processes. (a) STI first strained device. (b) and (c) STI last strained and relaxed devices.

\section{Device Characteristics}

The p-type Ge FinFETs for all evaluated STI processes have been fabricated at imec/Belgium on 300-mm Si (100) substrates. The device dimensions, such as fin width $\left(W_{\text {fin }}\right)$, geometric channel length $\left(L_{G}\right)$, fin height $\left(H_{\text {fin }}\right)$, number of fins in parallel $(N)$, gate-stack composition, and the Ge amount in the n-type in situ doped relaxed buffers, are presented in Table I. Moreover, the region underneath the channel has an n-type doping concentration around $5 \times 10^{18} \mathrm{~cm}^{-3}$. The processing details regarding the germanium layer growth for the three considered scenarios, i.e., STI last-strained, STI firststrained, and STI last-relaxed, can be found in [10]-[12], respectively, where Fig. 1 shows the main difference among them.

\section{Methodology}

This paper is based on extensive experimental data, where the LFN characterization has been performed in the linear operation regime, while the gate bias $\left(V_{\mathrm{GS}}\right)$ was stepped from weak to strong inversion from the hardware/software systems from ProPlusSolution. It is worth mentioning that the inputreferred voltage noise spectral density $\left(S_{\mathrm{VG}}\right)$ was determined from the ratio of the drain current noise spectral density over the square of the transconductance $\left(S_{\mathrm{ID}} / \mathrm{gm}^{2}\right)$. Regarding the input characteristic curves, the same operation regime has been used and obtained by an HP 4146C-Semiconductor Device Parameter Analyzer. The hole mobility and threshold voltage

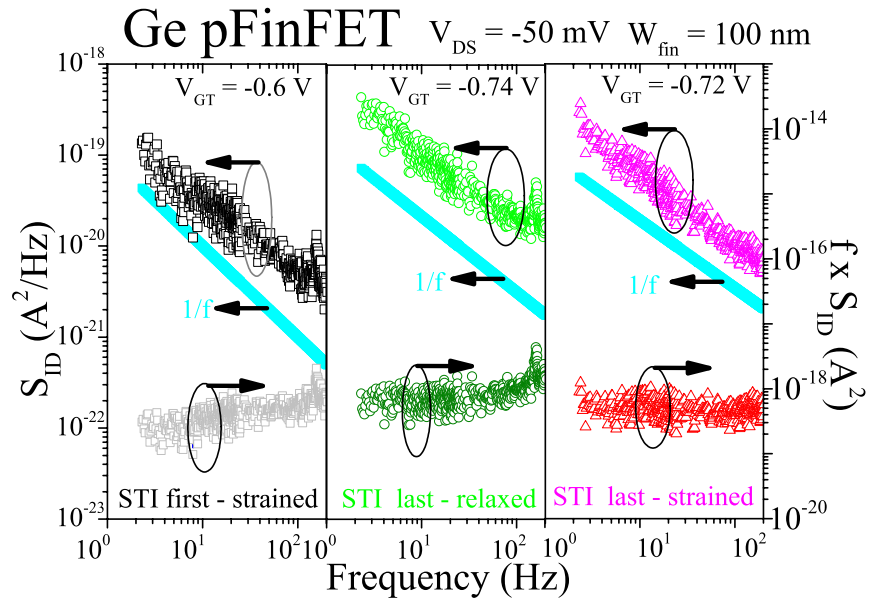

Fig. 2. LFN spectral density $\left(S_{\mathrm{ID}}\right)$ and $f \times S_{\mathrm{ID}}$ as a function of frequency in linear operation corresponding with planar-like $\left(W_{\text {fin }}=100 \mathrm{~nm}\right)$ devices for different STI processes.

were extracted by the $y$-function [17] and second derivative peak [18] methods, respectively.

The LFN is composed of three components as in (1), i.e., white noise, $1 / f^{\gamma}$ (flicker-noise), and the sum of Lorentzian components (generation-recombination: GR-noise) [19]. The first component is found in all spectra, on the other hand, it is negligible compared with other ones at frequencies below $10 \mathrm{kHz}$

$$
S_{\mathrm{VG}}(f)=B_{W}+\frac{K_{f}}{f^{\gamma}}+\sum_{i=0}^{N} \frac{A_{i}}{1+\left(\frac{f}{f_{0 i}}\right)^{2}}
$$

where $B_{W}$ is related to the white noise level, $k_{f} / f^{\gamma}$ presents the flicker noise, and $A_{i}$ and $f_{0 i}$ are the plateau value and characteristic frequency, respectively, of the different Lorentzian components.

The power spectral densities $\left(S_{\mathrm{ID}}\right)$ in Fig. 2 clearly show that the $1 / f^{\gamma}$ component is dominant for all STI processes. On top of that, the humps presented in $S_{\text {ID }}$ indicate the presence of a GR-noise component, which gives insights where the traps are located: either into the gate dielectric or into the channel region [20]. The GR-noise has been reported in detail in [21] taking into consideration both studied STI processes. Apart from that, the plateau behavior in the $f \times S_{\text {ID }}$ plots in Fig. 2 indicates that the $\gamma$ factor is close to one for all studied processes.

The $1 / f^{\gamma}$ can be influenced by two different mechanisms: carrier number fluctuation $(\Delta N)$ or mobility fluctuation $(\Delta \mu)$ or a correlation of both of them [22]. The dominant mechanism can be easily identified by checking whether there is a parallelism between the normalized power spectral density $\left(S_{\mathrm{ID}} / I_{\mathrm{DS}}^{2}\right)$ and the squared transconductance over drain current ratio $\left(\mathrm{gm} / \mathrm{I}_{\mathrm{DS}}\right)^{2}$ as a function of drain current [23]. If this is confirmed, the $\Delta \mathrm{N}$ is strongly predominant, otherwise $\Delta \mu$ fluctuations or an additional effect determine the $1 / f^{\gamma}$ noise.

Fig. 3 confirms the predominance of $\Delta N$ for the studied devices, similar as in Ge planar devices with different passivation layers [24], [25]. 


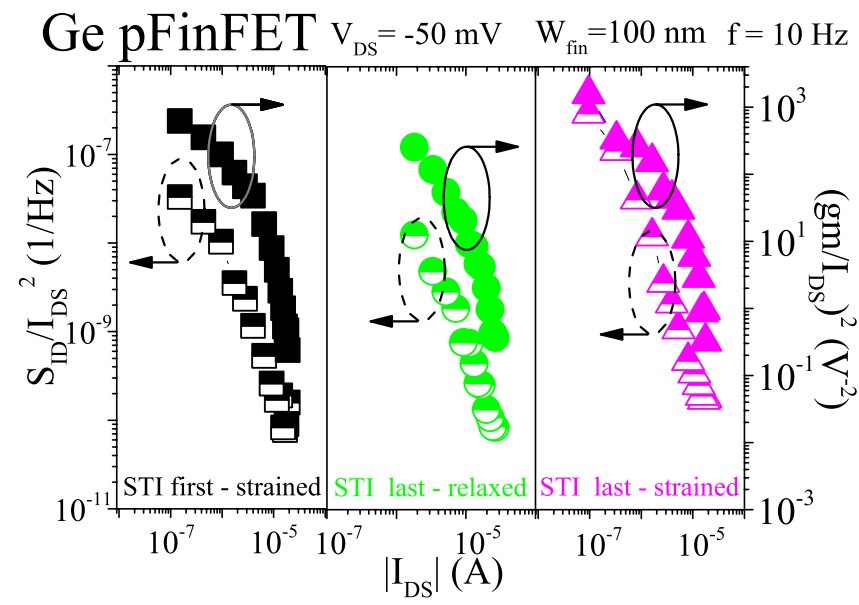

Fig. 3. Normalized drain current noise spectral density and $\left(\mathrm{gm} / \mathrm{I}_{\mathrm{DS}}\right)^{2}$ as a function of absolute drain current in linear operation for planar-like $\left(W_{\text {fin }}=100 \mathrm{~nm}\right)$ devices and different STI processes.

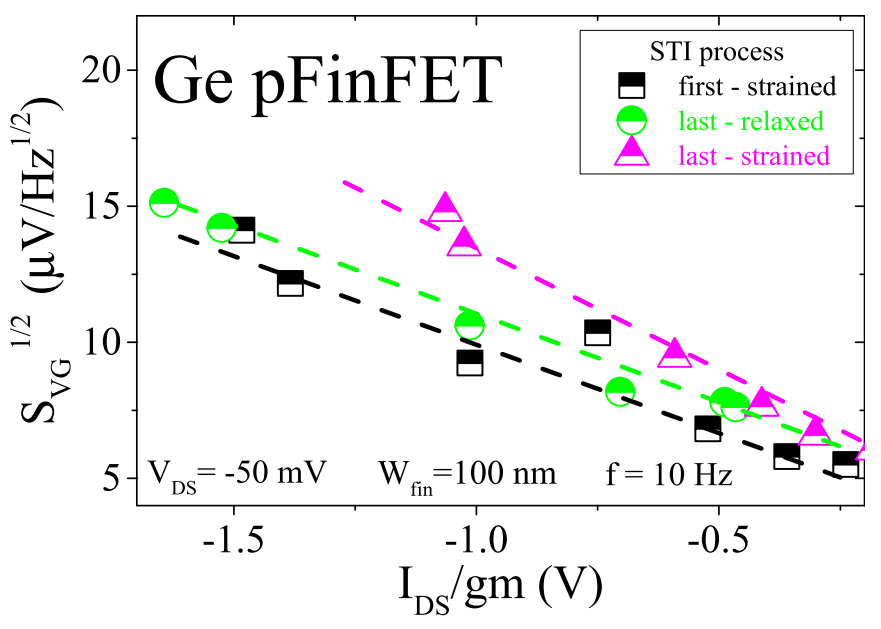

Fig. 4. Square root input-referred voltage noise spectral density in linear operation and $f=10 \mathrm{~Hz}$ as a function of drain current over transconductance ratio for planar-like $\left(W_{\text {fin }}=100 \mathrm{~nm}\right)$ devices and different STI processes.

Moreover, since $\Delta N$ is dominant, it allows the extraction of important parameters, such as Coulomb scattering coefficient $\left(\alpha_{\mathrm{sc}}\right)$, input-referred voltage noise at flat-band $\left(S_{\mathrm{VGfb}}\right)$, and oxide trap density $\left(N_{\mathrm{OT}}\right)$. The latter is determined by the following:

$$
S_{\mathrm{VGfb}}=\frac{q^{2} k_{B} \mathrm{TN}_{\mathrm{OT}}}{W_{\mathrm{eff}} L_{G} \alpha_{t} C_{\mathrm{OX}}^{2} f}
$$

where $q$ is the elementary charge, $k_{B} T$ is the thermal energy, $W_{\text {eff }}$ is the effective width, $L_{G}$ is the geometric channel length, $f$ is the frequency, $\alpha_{t}$ is the attenuation tunneling parameter noise taken as $1 \times 10^{8} \mathrm{~cm}^{-1}$, and $C_{\mathrm{OX}}$ is the capacitance density.

In order to extract $N_{\mathrm{OT}}$, both $\alpha_{\mathrm{sc}}$ and $S_{\mathrm{VGfb}}$ values must be known and can be obtained from (3). By plotting this equation as done in Fig. $4, \alpha_{\mathrm{sc}}$ and $S_{\mathrm{VGfb}}$ are determined from the intercept and slope of the curves. By assuming an energy interval of $4 k_{B} T, N_{\mathrm{OT}}\left(\mathrm{eV}^{-1} \mathrm{~cm}^{-3}\right)$ can be converted into surface density $\left(D_{\mathrm{OT}}\right)[26]$, considering a thickness of the

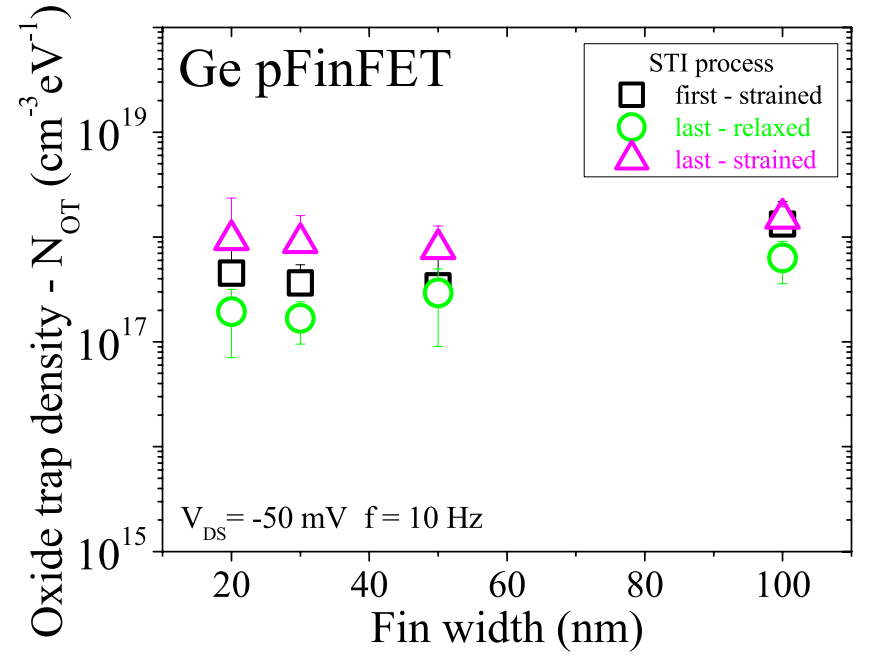

Fig. 5. Oxide trap density as a function of fin width for different STI processes.

trapping-detrapping layer of $2 \mathrm{~nm}$

$$
S_{\mathrm{VG}}=S_{\mathrm{VGfb}}\left(1+\alpha_{\mathrm{SC}} \mu C_{\mathrm{OX}} \frac{I_{\mathrm{DS}}}{\mathrm{gm}}\right)^{2}
$$

where $S_{\mathrm{VG}}$ is the input-referred voltage noise spectral density and $\mu$ is the mobility.

The Coulomb scattering $\left(\alpha_{\mathrm{SC}}\right)$ can also be extracted from the linear dependence of the inverse mobility $(1 / \mu)$ with $D_{\mathrm{OT}}$, as shown in [27]

$$
\frac{1}{\mu}=\frac{1}{\mu_{0}}+q D_{\mathrm{OT}} \alpha_{\mathrm{SC}}
$$

where $\mu_{0}$ represents the mobility limited by other scattering mechanisms apart from the Coulomb scattering of the oxide.

\section{REsults}

Fig. 5 shows the oxide trap density ( $\left.N_{\mathrm{OT}}\right)$ and reveals that for Ge FinFETs, the $N_{\mathrm{OT}}$ level is around $10^{17} \sim 10^{18} \mathrm{~cm}^{-3} \mathrm{eV}^{-1}$, which is lower than for planar Ge MOSFET devices, which is around $5 \times 10^{18} \mathrm{~cm}^{-3} \mathrm{eV}^{-1} \sim$ $3 \times 10^{19} \mathrm{~cm}^{-3} \mathrm{eV}^{-1}$ [25], [27] and similar to values for $\mathrm{Si}$ FinFETs with a similar gate-stack [28]-[30].

Fig. 6 presents the hole mobility $\left(\mu_{h}\right)$ for different fin widths, and in STI last strained devices, no noticeable $W_{\text {fin }}$ dependence is observed. On the other hand, the other STI processes show a hole mobility increase, when the fin width reduces, because of the strong sidewall contribution, since the low-field hole mobility of the FinFET on the top $\langle 100\rangle$ plane is inferior over the value for $\langle 110\rangle$ sidewalls [31]. It is also clear in Fig. 6 that for the STI first strained devices, the compressive stress has only a marginal impact on the hole mobility, while there is a clear improvement for the STI last strained counterparts. As shown in the following, this is related with a more pronounced impact of Coulomb scattering in the former case.

Fig. 7 presents the Coulomb scattering coefficient $\left(\alpha_{\mathrm{SC}}\right)$ and clearly shows the one order of magnitude higher value for STI first compared with both STI last processes, which might play 


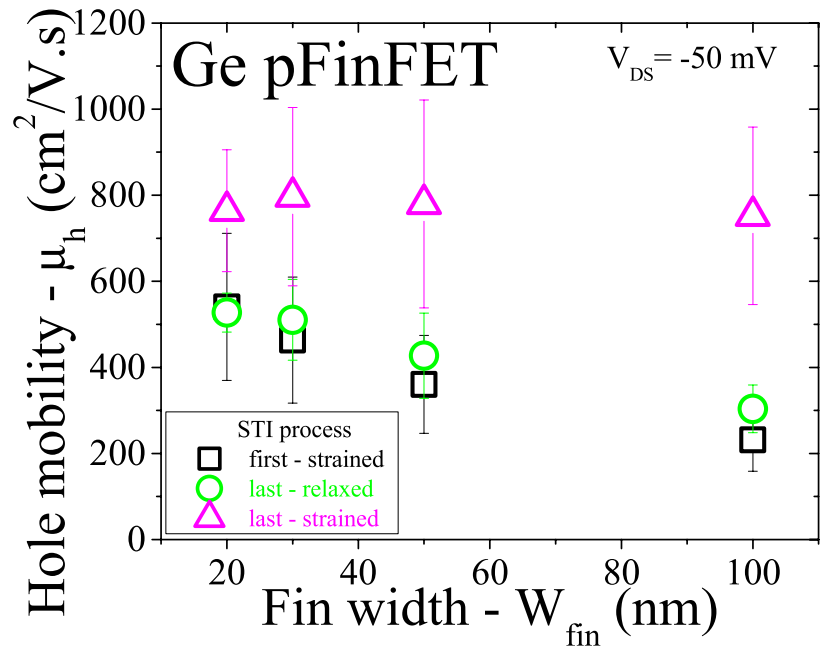

Fig. 6. Hole mobility as a function of fin width for different STI processes.

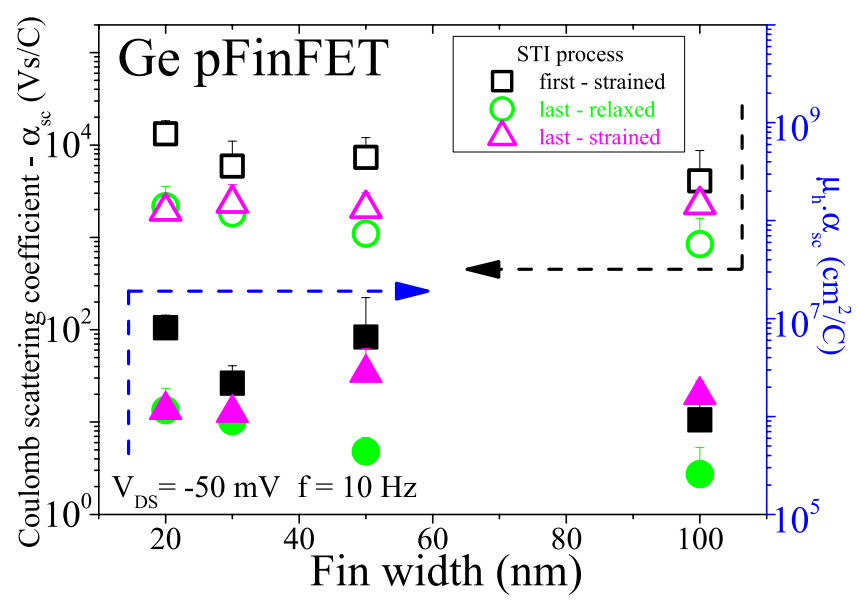

Fig. 7. Coulomb scattering coefficient as a function of fin width for different STI processes.

a role in the device performance, due to its impact on the hole mobility. The obtained values are similar to those for Ge pMOSFETs, which is between $10^{3} \sim 10^{4} \mathrm{Vs} / \mathrm{C}$ [25], [27]. Moreover, a slight fin width $\left(W_{\text {fin }}\right)$ dependence is found, except for the STI last strained process where there is no correlation between $\alpha_{\mathrm{SC}}$ and $W_{\text {fin }}$ and its $\alpha_{\mathrm{SC}}$ value is around $2 \times 10^{3} \mathrm{Vs} / \mathrm{C}$.

A higher scattering coefficient indicates more efficient scattering by charged traps, which are on the average closer to the channel. It implies that the charge centers in the STI first case are closer to the inversion layer, either in the gatestack or perhaps, more directly in the Ge fin. From the product of $\mu_{h}$ and $\alpha_{\mathrm{SC}}$, which is presented in a secondary $y$-axis in Fig. 7, one can derive that the STI first approach experiences the strongest effect of the Coulomb scattering by charged oxide traps, probed by the 1/f noise PSD.

Fig. 8 shows the influence of fin width on the threshold voltage $\left(V_{T}\right)$, where there is both a $W_{\text {fin }}$-dependence and strain effect. The latter results in a $V_{T}$ difference between strain and relaxed devices $\left(\Delta V_{T}\right)$ of about $0.3 \mathrm{~V}$, as a consequence of the Ge valence band $\left(E_{V}\right)$ shifting when compressive strain is applied in p-channel $\mathrm{Ge}$ devices, i.e., there is a Ge bandgap

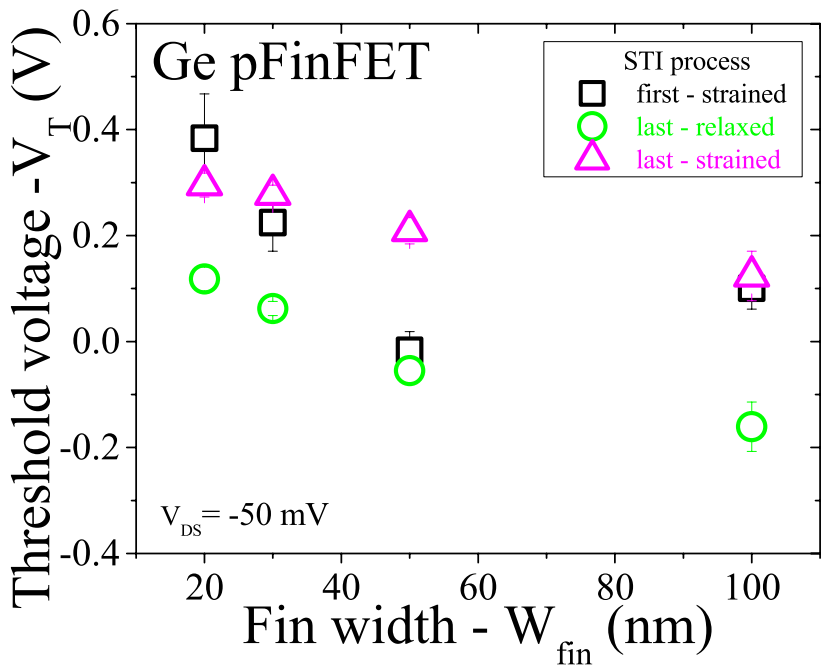

Fig. 8. Threshold voltage as a function of fin width for different STI processes.

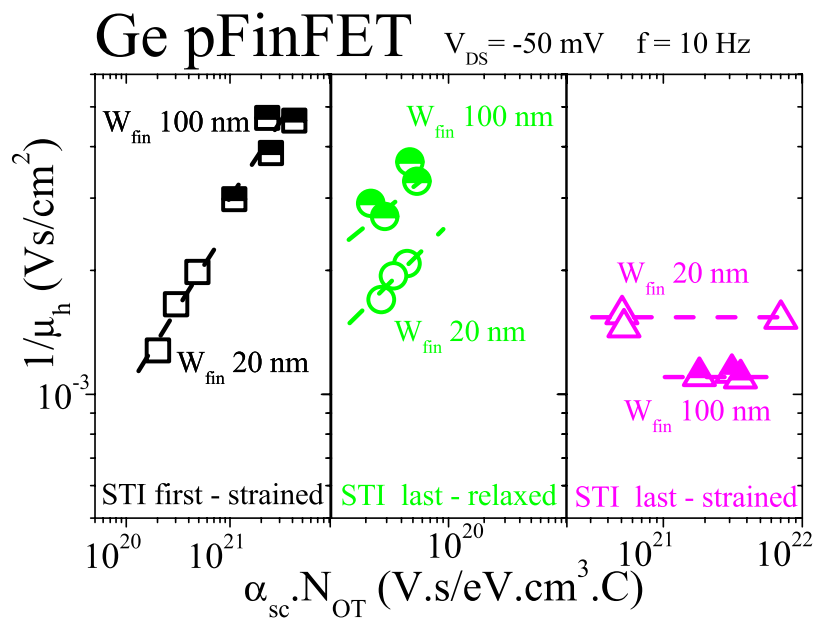

Fig. 9. Inverse hole mobility as a function of scattering coefficient times oxide trap density for different STI processes.

reduction [32]. The $W_{\text {fin }}$-dependence might be associated with the doping concentration that can be inferior to narrow devices compared with planar-like ones.

\section{DISCUSSION}

Fig. 9 uses the product of the Coulomb scattering coefficient and oxide trap density to evaluate their influence on the hole mobility $\left(\mu_{h}\right)$.

The more pronounced $\mu_{h}$ degradation is found for STI first strained narrow and planar-like devices, indicating that the Coulomb scattering mechanism might be dominant. The STI last relaxed devices also present $\mu_{h}$ degradation, however, less prominent than for the STI first ones. On the other hand, $\mu_{h}$ shows no clear correlation with $\alpha_{\mathrm{SC}}$ times $N_{\mathrm{OT}}$ for the STI last strained process.

Fig. 10 presents the impact of the oxide trap density on the threshold voltage. There is a slight variation of the $V_{T}$ value for STI last strained narrow devices, resulting in a lower $V_{T}$ value compared with STI first devices, as presented in Fig. 8 ( $W_{\text {fin }}$ of $20 \mathrm{~nm}$ ). For both STI first and last processes, strained 


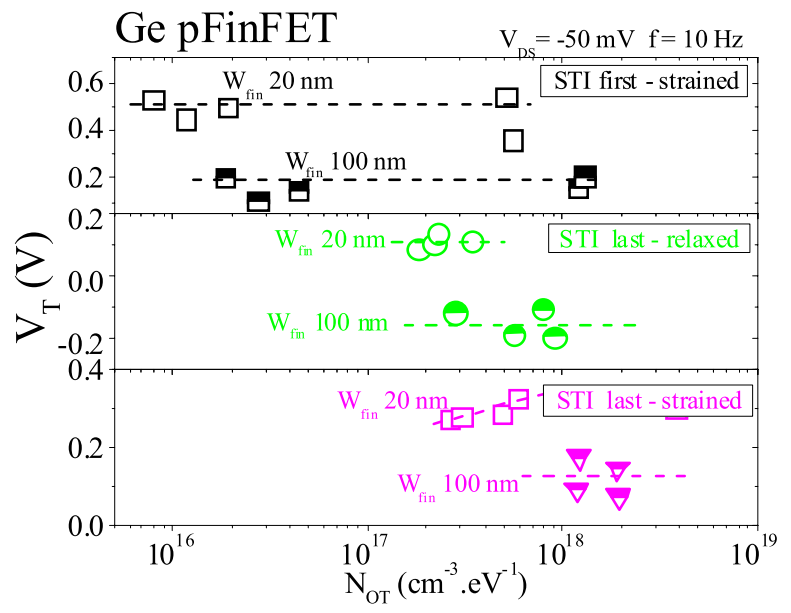

Fig. 10. Threshold voltage as a function of oxide trap density for different STI processes and two fin widths.

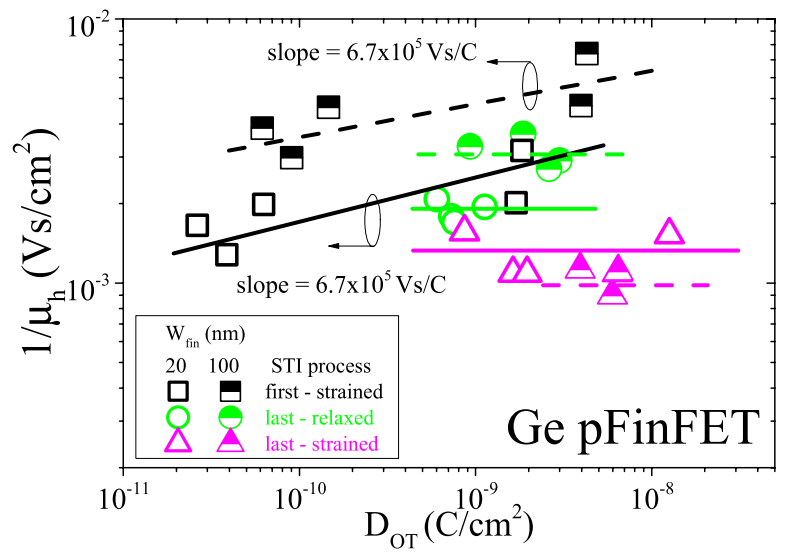

Fig. 11. Inverse hole mobility as a function of surface trap charge density for different STI processes.

and relaxed, there is no clear correlation that might affect the $V_{T}$ behavior.

Fig. 11 presents the inverse hole mobility as a function of surface trap charge density. Despite the fact that the Coulomb scattering coefficient obtained from Fig. 11 $\left(6.7 \times 10^{5} \mathrm{Vs} / \mathrm{C}\right)$ is about one order of magnitude higher than the ones from Fig. 7 (STI first process). It gives further support to the predominance of the Coulomb scattering mechanism independent of the fin width only for the STI first process, which has also been demonstrated by low-temperature split$\mathrm{CV}$ measurements [33]. It might be associated with fixed charges into the gate-stack layer [34].

The latter presents thinner and thicker layers of $\mathrm{SiO}_{2}$ and $\mathrm{HfO}_{2}$, respectively, for STI first process compared with the STI last ones, indicating that thicker high- $k$ layer might contribute to the predominance of the Coulomb scattering mechanisms. This is supported by the higher $\alpha_{\mathrm{SC}}$ values in Fig. 7. A thinner interfacial $\mathrm{SiO}_{2}$ layer brings the charged traps in the $\mathrm{HfO}_{2}$ closer to the channel, resulting in more efficient Coulomb scattering.

\section{CONCLUSION}

For the studied Ge pFinFETs, the dominant noise mechanism, independent of the used STI process, is number fluctuations. Furthermore, the STI last processes demonstrate to be less affected by the Coulomb scattering, at least for strained devices. In contrast, the Coulomb scattering mechanism plays an important role for STI first strained devices, resulting in a degradation of the hole mobility. The strain effect has only a pronounced impact on the threshold voltage and not on the hole mobility, indicating that there are other mechanisms playing a role. Finally, the oxide trap density can degrade the device $V_{T}$, although the STI first-strained and last-relaxed approaches show no significant correlation between these parameters.

\section{ACKNOWLEDGMENT}

The authors would like to thank CAPES, FWO, and the Logic IIAP Program for the support.

\section{REFERENCES}

[1] S. Takagi and M. Takenaka, "Prospective and critical issues of III-V/Ge CMOS on Si platform," Electrochem. Soc. Trans., vol. 35, no. 3, pp. 279-298, May 2011, doi: 10.1149/1.3569921.

[2] T. Chiarella et al., "Benchmarking SOI and bulk FinFET alternatives for PLANAR CMOS scaling succession," Solid-State Electron., vol. 54, no. 9, pp. 855-860, Sep. 2010, doi: 10.1016/j.sse.2010. 04.010.

[3] H. Iwai, "Future of nano CMOS technology," Solid-State Electron., vol. 112, pp. 56-57, Oct. 2015, doi: 10.1016/j.sse.2015. 02.005 .

[4] S. Takagi et al., "High mobility CMOS technologies using III-V/Ge channels on Si platform," Solid-State Electron., vol. 88, pp. 2-8, Oct. 2013, doi: 10.1016/j.sse.2013.04.020.

[5] J. Robertson and R. M. Wallace, "High- $K$ materials and metal gates for CMOS applications," Mater. Sci. Eng. R, Rep., vol. 88, pp. 1-41, Feb. 2015, doi: 10.1016/j.mser.2014.11.001.

[6] R. People and J. C. Bean, "Calculation of critical layer thickness versus lattice mismatch for $\mathrm{Ge}_{x} \mathrm{Si}_{1-x} / \mathrm{Si}$ strained-layer heterostructures," Appl. Phys. Lett., vol. 47, no. 3, pp. 322-324, Aug. 1985, doi: 10.1063/1.96206.

[7] E. Simoen et al., "Challenges and opportunities in advanced Ge pMOSFETs," Mater. Sci. Semicond. Process., vol. 15, no. 6, pp. 588-600, Dec. 2012, doi: 10.1016/j.mssp.2012.04.017.

[8] E. Simoen et al., "Is there an impact of threading dislocations on the characteristics of devices fabricated in strained-Ge substrates?" Phys. Status Solidi C, vol. 6, no. 8, pp. 1912-1917, Aug. 2009, doi: $10.1002 / \mathrm{pssc} .200881446$.

[9] E. Simoen et al., "High doping density/high electric field, stress and heterojunction effects on the characteristics of CMOS compatible p-n junctions," J. Electrochem. Soc., vol. 158, no. 5, pp. R27-R36, Mar. 2011, doi: 10.1149/1.3555103.

[10] R. Loo et al., "Smooth and high quality epitaxial strained Ge grown on SiGe strain relaxed buffers with 70-85\% Ge," J. Crystal Growth, vol. 324, no. 1, pp. 15-21, Jun. 2011, doi: 10.1016/j.jcrysgro.2011.03.030.

[11] R. Loo et al., "High quality Ge virtual substrates on $\mathrm{Si}$ wafers with standard STI patterning," J. Electrochem. Soc., vol. 157, no. 1, pp. H13-H21, Nov. 2009, doi: 10.1149/1.3244564.

[12] L. Witters et al., "Strained germanium quantum well pMOS FinFETs fabricated on in situ phosphorus-doped SiGe strain relaxed buffer layers using a replacement Fin process," in Proc. IEEE Int. Electron Devices Meeting, Washington, DC, USA, Dec. 2013, pp. 20.4.1-20.4.4, doi: 10.1109/IEDM.2013.6724669.

[13] J. Z. Li, J. Bai, C. Major, M. Carroll, A. Lochtefeld, and Z. Shellenbarger, "Defect reduction of GaAs/Si epitaxy by aspect ratio trapping," J. Appl. Phys., vol. 103, no. 10, p. 106102, 2008, doi: 10.1063/1.2924410.

[14] M. T. Currie, S. B. Samavedam, T. A. Langdo, C. W. Leitz, and E. A. Fitzgerald, "Controlling threading dislocation densities in $\mathrm{Ge}$ on Si using graded SiGe layers and chemical-mechanical polishing," Appl. Phys. Lett., vol. 72, pp. 1718-1720, Apr. 1998, doi: 10.1063/ 1.121162 . 
[15] G. Nicholas et al., "High mobility strained Ge pMOSFETs with high- $k$ /metal gate," IEEE Electron Device Lett., vol. 28, no. 9, pp. 825-827, Sep. 2007, doi: 10.1109/LED.2007.903405.

[16] C. Claeys and E. Simoen, "Noise as a diagnostic tool for semiconductor material and device characterization," J. Electrochem. Soc., vol. 145, no. 6, pp. 2058-2067, Jan. 1998, doi: 10.1149/1.1838597.

[17] G. Ghibaudo, "New method for the extraction of MOSFET parameters," Electron. Lett., vol. 24, no. 9, pp. 543-545, Apr. 1988, doi: 10.1049/el:19880369.

[18] L. Dobrescu, M. Petrov, D. Dobrescu, and C. Ravariu, "Threshold voltage extraction methods for MOS transistors," in Proc. Int. Semicond. Conf., Sinaia, Romania, 2000, pp. 371-374, doi: 10.1109/SMICND.2000.890257.

[19] S. D. dos Santos et al., "Low-frequency noise assessment in advanced UTBOX SOI nMOSFETs with different gate dielectrics," Solid-State Electron., vol. 97, pp. 14-22, Jul. 2014, doi: 10.1016/j.sse.2014.04.034.

[20] I. Lartigau et al., "Low temperature noise spectroscopy of $0.1 \mu \mathrm{m}$ partially depleted silicon on insulator metal-oxide-semiconductor field effect transistors," J. Appl. Phys., vol. 101, no. 10, p. 104511, May 2007, doi: $10.1063 / 1.2732685$.

[21] A. V. Oliveira et al., "GR-noise characterization of Ge pFinFETs with STI first and STI last processes," IEEE Electron Device Lett., to be published, doi: 10.1109/LED.2016.2595398.

[22] E. Simoen and C. Claeys, "On the flicker noise in submicron silicon MOSFETs," Solid-State Electron., vol. 43, no. 5, pp. 865-882, May 1999, doi: 10.1016/S0038-1101(98)00322-0.

[23] G. Ghibaudo, O. Roux, C. Nguyen-Duc, F. Balestra, and J. Brini, "Improved analysis of low frequency noise in field-effect MOS transistors," Phys. Status Solidi A, vol. 124, no. 2, pp. 571-581, Apr. 1991, doi: $10.1002 /$ pssa.2211240225.

[24] W. Fang et al., "Low-frequency noise study of Ge p-MOSFETs with $\mathrm{HfO}_{2} / \mathrm{Al}_{2} \mathrm{O}_{3} / \mathrm{GeO}_{x}$ gate stack," in Proc. Int. Conf. Noise Fluctuations, Xian, China, Jun. 2015, pp. 1-4, doi: 10.1109/ICNF.2015.7288550.

[25] W. Guo et al., "Low-frequency noise assessment of silicon passivated $\mathrm{Ge}$ pMOSFETs with TiN/TaN/ $\mathrm{HfO}_{2}$ gate stack," IEEE Electron Device Lett., vol. 28, no. 4, pp. 288-291, Apr. 2007, doi: 10.1109/LED.2007.891797.

[26] G. Ghibaudo and T. Boutchacha, "Electrical noise and RTS fluctuations in advanced CMOS devices," Microelectron. Rel., vol. 42, nos. 4-5, pp. 573-582, Apr./May 2002, doi: 10.1016/S0026-2714(02)00025-2.

[27] W. Fang et al., "Low-frequency noise characterization of $\mathrm{GeO}_{x}$ passivated germanium MOSFETs," IEEE Trans. Electron Devices, vol. 62, no. 7, pp. 2078-2083, Jul. 2015, doi: 10.1109/TED.2015.2430367.

[28] M. G. C. de Andrade, J. A. Martino, E. Simoen, and C. Claeys, "Comparison of the low-frequency noise of bulk triple-gate FinFETs with and without dynamic threshold operation," IEEE Electron Device Lett., vol. 32, no. 11, pp. 1597-1599, Nov. 2011, doi: 10.1109/LED.2011.2166372.

[29] E. Simoen, J.-W. Lee, A. Veloso, V. Paraschiv, N. Horiguchi, and C. Claeys, "Low-frequency-noise-based oxide trap profiling in replacement high- $k$ /metal-gate pMOSFETs," ECS J. Solid State Sci. Technol., vol. 3, no. 6, pp. Q127-Q-131, May 2014, doi: 10.1149/2.017406jss.

[30] J.-S. Lee, Y.-K. Choi, D. Ha, T.-J. King, and J. Bokor, "Lowfrequency noise characteristics in p-channel FinFETs," IEEE Electron Device Lett., vol. 23, no. 12, pp. 722-724, Dec. 2002, doi: 10.1109/LED.2002.805741.

[31] I.-H. Wong, Y.-T. Chen, J.-Y. Yan, H.-J. Ciou, Y.-S. Chen, and C.-W. Liu, "Fabrication and low temperature characterization of Ge (110) and (100) p-MOSFETs," IEEE Trans. Electron Devices, vol. 61, no. 6, pp. 2215-2219, Jun. 2014, doi: 10.1109/TED.2014.2318083.

[32] R. Kim, U. E. Avci, and I. A. Young, "CMOS performance benchmarking of $\mathrm{Si}$, InAs, GaAs, and Ge nanowire n- and pMOSFETs with $\mathrm{Lg}=13 \mathrm{~nm}$ based on atomistic quantum transport simulation including strain effects," in Proc. IEEE Int. Electron Devices Meeting, Washington, DC, USA, Dec. 2015, pp. 34.1.1-34.1.4, doi: 10.1109/IEDM.2015.7409824.

[33] A. V. Oliveira et al., "Split-CV mobility at low temperature operation on Ge pFinFETs fabricated with STI first and last processes," Semicond. Sci. Technol., to be published.

[34] B. Ghosh, J.-H. Chen, X.-F. Fan, L. F. Register, and S. K. Banerjee, "Monte Carlo study of remote Coulomb and remote surface roughness scattering in nanoscale Ge PMOSFETs with ultrathin high- $k$ dielectrics," Solid-State Electron., vol. 50, no. 2, pp. 248-253, Feb. 2006, doi: 10.1016/j.sse.2005.12.007.

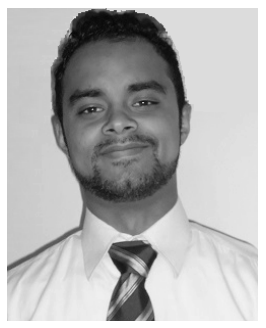

Alberto V. de Oliveira (S'14) received the B.Sc. degree in electrical engineering from the Centro Universitário da FEI, São Bernardo do Campo, Brazil, in 2012. He is currently pursuing the $\mathrm{Ph} . \mathrm{D}$. degree in microelectronics with the University of São Paulo, São Paulo, Brazil.

$\mathrm{He}$ is an International Scholar with Katholieke Universiteit Leuven and imec, Leuven, Belgium.

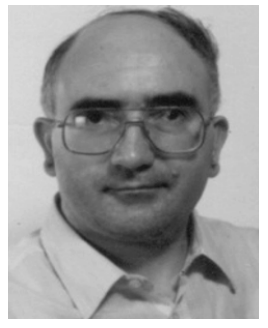

Eddy Simoen (M'16) received the M.S. and $\mathrm{Ph} . \mathrm{D}$. degrees in engineering from Ghent University, Ghent, Belgium, in 1980 and 1985, respectively.

$\mathrm{He}$ has been with imec, Leuven, Belgium, since 1986, where he is currently a Scientist.

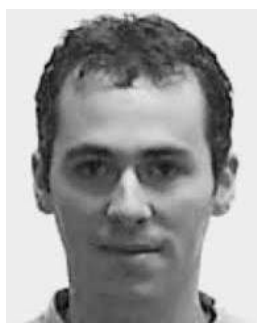

Jerome Mitard received the Ph.D. degree in microelectronics from the Laboratory of Electronic Information Technology, Grenoble, France, and STMicroelectronics, Grenoble.

He joined imec, Leuven, Belgium, as a Device Researcher in 2007, where he has been involved in high-mobility channel MOSFETs. He is currently the Team Leader of the $300 \mathrm{~mm}$ Platform Device Research Team.

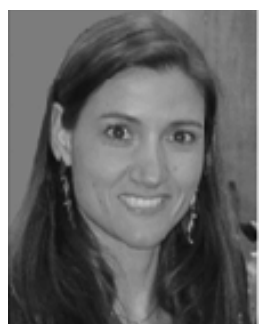

Paula G. D. Agopian (M'09-SM'15) received the M.S. and Ph.D. degrees in microelectronics from the University of São Paulo (USP), São Paulo, Brazil.

She was a Researcher and an IC Project Manager of the Training Center with USP. She is currently a Professor with Universidade Estadual Paulista, São João da Boa Vista, Brazil.

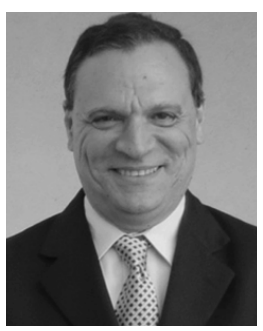

Joao Antonio Martino (M'06-SM'07) received the M.S., and Ph.D. degrees in microelectronic from the University of São Paulo (USP), São Paulo, Brazil.

$\mathrm{He}$ held a post-doctoral position with imec, Leuven, Belgium on SOI MOSFET devices. He has been Professor with USP since 2005 and he has been involved in UTBB SOI, FinFET, and Tunnel-FET devices.

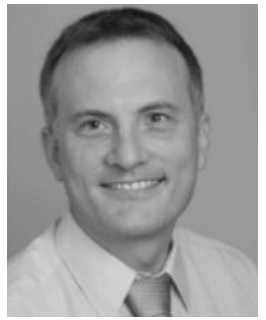

Robert Langer received the Dipl.-Physiker degree from Universität Karlsruhe, Karlsruhe, Germany, in 1996, and the Ph.D. degree from Université Joseph Fourier, Grenoble, France, in 2000.

$\mathrm{He}$ has been with imec, Leuven, Belgium, since 2014, as R\&D Manager of the Epitaxial Growth Group. 


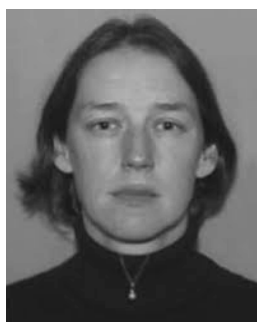

Liesbeth Witters received the B.Sc. degree in chemical engineering from Katholieke Universiteit Leuven, Leuven, Belgium, in 1992, and the M.Sc. degree in chemical engineering from the Institut Frans du Pole, Paris, France, in 1993.

She joined imec, Leuven, in 2001, where she is currently involved in CMOS process development.

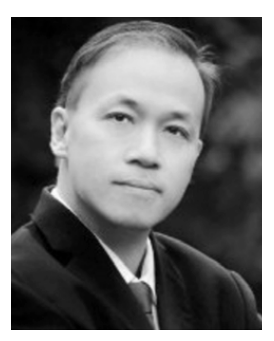

Aaron Voon-Yew Thean (SM'15) received the B.Sc., M.Sc., and Ph.D. degrees from the University of Illinois Urbana-Champaign, Urbana, IL, USA, all in electrical engineering.

He was the Director of the Logic Program with imec, Leuven, Belgium. He is currently a Professor with the National University of Singapore, Singapore.

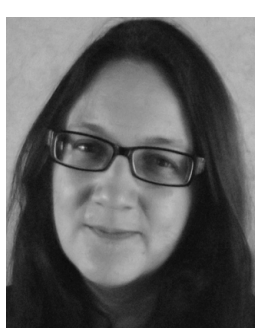

Nadine Collaert received the M.S. and $\mathrm{Ph} . \mathrm{D}$. degrees in electrical engineering from the Katholieke Universiteit Leuven, Leuven, Belgium, in 1995 and 2000, respectively.

She has been a Program Manager of the LOGIC Program with imec, Leuven, since 2012, focusing on high-mobility channels, TFETs, and nanowires.

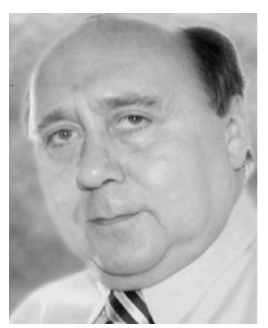

Cor Claeys (M'94-SM'95-F'09) received the $\mathrm{Ph} . \mathrm{D}$. degree from Katholieke Universiteit Leuven (KU Leuven), Leuven, Belgium.

$\mathrm{He}$ has been a Professor with KU Leuven since 1990. He has also been with imec, Leuven, since 1984 